Article

\title{
Single Pass Laser Process for Super-Hydrophobic Flexible Surfaces with Micro/Nano Hierarchical Structures
}

\author{
Hyuk-Jun Kwon ${ }^{1}$, Junyeob Yeo ${ }^{2} \mathbb{D}^{\mathbb{C}}$, Jae Eun Jang ${ }^{1}$, Costas P. Grigoropoulos ${ }^{3, *}$ and \\ Jae-Hyuck Yoo ${ }^{3,4, *}$ \\ 1 Department of Information and Communication Engineering, DGIST, Daegu 42988, Korea; \\ hj.kwon@dgist.ac.kr (H.-J.K.); jang1@dgist.ac.kr (J.E.J.) \\ 2 Department of Physics, Kyungpook National University, Daegu 41566, Korea; junyeob@knu.ac.kr \\ 3 Department of Mechanical Engineering, University of California, Berkeley, CA 94720, USA \\ 4 Physical and Life Sciences and NIF and Photon Sciences, Lawrence Livermore National Laboratory, \\ Livermore, CA 94550, USA \\ * Correspondence: cgrigoro@me.berkeley.edu (C.P.G.); yoo5@llnl.gov (J.-H.Y.)
}

Received: 25 June 2018; Accepted: 12 July 2018; Published: 17 July 2018

\begin{abstract}
Wetting has been studied in various fields: chemical industry, automobile manufacturing, food companies, and even life sciences. In these studies, super-hydrophobic surfaces have been achieved through complex steps and processes. To realize super-hydrophobicity, however, we demonstrated a simple and single pass laser process for the fabrication of micro/nano hierarchical structures on the flexible polytetrafluoroethylene (PTFE, Teflon) surface. The fabricated hierarchical structures helped increase the hydrophobicity by augmenting the surface roughness and promoting air-trapping. In addition, we employed a low-cost and high-throughput replication process producing numerous polydimethylsiloxane (PDMS) replicas from the laser-processed PTFE film. Thanks to the anti-adhesive characteristics of PTFE and the elasticity of PDMS, the structure perfectly transferred to the replica without any mechanical failure. Moreover, our designed mesh patterns offered the possibility of large area applications through varying the process parameters (pitch, beam spot size, laser fluence, and scan speed). Even though mesh patterns had relatively large pitch $(190 \mu \mathrm{m})$, we were able to achieve high contact angle $\left(>150^{\circ}\right)$. Through pneumatically deformed structure, we clearly showed that the shape of the droplets on our laser-processed super-hydrophobic surface was spherical. Based on these outcomes, we can expect our single laser pulse exposure process can overcome many drawbacks and offer opportunities for advancing applications of the wetting phenomena.
\end{abstract}

Keywords: super-hydrophobic; laser process; polytetrafluoroethylene (PTFE); polydimethylsiloxane (PDMS); hierarchical structures

\section{Introduction}

One can observe water droplets rolling off the surface of lotus leaves during rain. This hydrophobicity typically comes from the roughness of tiny micro-scale structures and water repellent materials. Depending on the surface condition (e.g., material and roughness) and tilt angle, droplets on the surface experience different accelerations as they are subject to gravity and/or airflow drag. Research on wettability of a solid surface and the water contact angle has been widely explored in industry and in academia through the years. The models of Wenzel (where the droplet is completely wet between microstructures) and Cassie (that assumes air is trapped between these micro- structures while the droplet stays on top of the microstructures and trapped air) are usually employed for describing the relation between the surface structure and hydrophobicity. To improve the lotus effect 
(i.e., reduce friction resistance and adhesion force), artificial super-hydrophobic surfaces characterized by water contact angle larger than $150^{\circ}$ have attracted great attention in fundamental research and practical applications [1-3]. Various approaches have been reported by controlling the chemical composition [4-6] by fabricating micro/nano structures [7,8], modifying the physical morphology of the surface [9], and using water repellent materials [1-3,7]. Through the control of these surface characteristics, various useful practical applications are demonstrated, such as self-cleaning [10-12], anti-icing [13,14], water harvesting [15], no loss transport [16,17], and electrowetting [18,19]. However, due to technical issues, implementations have been limited. For example, to achieve the super-hydrophobic surface, complicated multiple steps are typically required. Also, these techniques cannot easily form hierarchical micro/nano structures promoting hydrophobicity via increasing the multiscale surface roughness. In this study, we directly wrote super-hydrophobic surfaces with nano and micro hierarchical structures on PTFE (McMaster-Carr, Elmhurst, IL, USA, Adhesive-Backed film) by single femtosecond (fs) laser pulse irradiation. Femtosecond laser (particularly single pass process) is used for industrial applications: mechanical testing [20] and tuning optical properties [21].

Super-hydrophobic process producing numerous polydimethylsiloxane (PDMS) (Sylgard 184, Dow Corning, Midland, MI, USA) surface was achieved by replication. Hierarchical polymer surfaces could be duplicated many times. In addition, we designed a specific mesh pattern for the scaling up of the effective area through parametric study. The results will be helpful for using pulsed laser processing to modify the wettability of the surface and for understanding the interaction between various micro/nano hierarchical structures and the water droplets. Also, we demonstrated droplet position control on pneumatically modified flexible super-hydrophobic surface where droplets easily move at a small tilt angle.

\section{Result and Discussion}

Figure 1a shows scanning electron microscopy (SEM) images of the PTFE surface upon single laser pulse irradiation at varied laser fluences. Here, we note that PTFE offers very attractive physical and chemical properties: low friction resistance, low surface adhesion, thermal and chemical stability, biocompatible, and high water repellency $[5,6]$. These physical and chemical features allow formation of fiber-like micro/nano hierarchical structures unlike any other polymers (e.g., polyimide). This is unique phenomenon to PTFE offering a great advantage for easily generating super-hydrophobic surface through just a single femtosecond (fs) laser shot. Furthermore, all laser processes in this study were carried out with laser (Spitfire, Spectra-physics, Santa Clara, CA, USA) of 100 femtosecond pulse duration, $400 \mathrm{~nm}$ wavelength, and $1 \mathrm{kHz}$ repetition rate. The laser system was equipped with the XY translation stage (ANT130, Aerotech Inc., Clovis, NM, USA), a laser power attenuator (consisting of a half wave plate and a polarizing beam splitter), a mechanical shutter (Thorlabs, Newton, NJ, USA, $\mathrm{SC} 10)$, and in-situ monitoring system. For focusing the laser beam, long working distance objective lenses (Mitutoyo, Takatsu ku, Japan) were used. In Figure 1a, ablative removal of PTFE material was observed in the center, resulting in a concave surface profile as presented with the black line. In addition, fibrous debris (i.e., the explosive ejection of molten PTFE material) was radially distributed along the perimeter during the laser ablation process [20]. Since PTFE exhibits its first linear absorption peak at $160 \mathrm{~nm}$ and the wavelength of the laser used in this study is $400 \mathrm{~nm}$ with 100 femtosecond (fs) pulse duration, laser energy should be absorbed by multi-photon process [22,23]. Figure $1 \mathrm{~b}$ shows the squared diameter $D^{2}$ of the ablated areas that is linearly proportional to the logarithm of the incident laser fluence. With $1 / e^{2}$ laser beam spot radius $\left(\mathrm{w}_{o}=6.44 \mu \mathrm{m}\right)$, the ablation threshold $\left(\Phi_{t h}\right)$ is approximately calculated as $0.77 \mathrm{~J} / \mathrm{cm}^{2}$ based on $D^{2}=2 w_{0}^{2} \ln \left(\Phi_{0} / \Phi_{t h}\right)$, where $\Phi_{o}$ is the average laser fluence that is defined as the pulse energy $(E)$ per irradiated area, $\Phi_{0}=E /\left(\pi w_{0}{ }^{2}\right)$ [24]. In a low-cost and high-throughput process, this fast laser direct writing (LDW) is used as the master mold fabrication. Furthermore, laser assisted PTFE master molds with micro/nano hierarchical structures have been utilized to generate replicas on the surface of PDMS. Figure 1c shows SEM pictures of PDMS replicas from the ablated PTFE film, having convex surface profile as illustrated by the black line. 


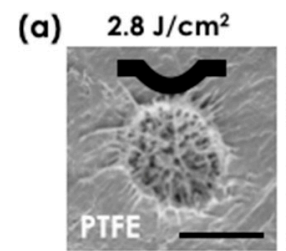

(c)

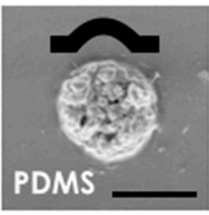

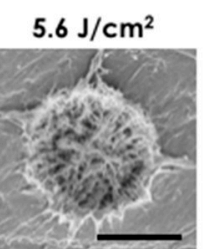

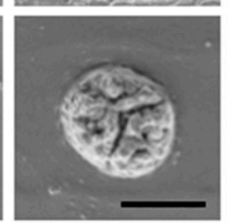

$9.8 \mathrm{~J} / \mathrm{cm}^{2}$
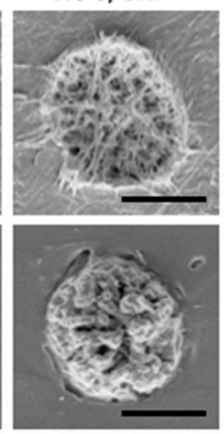

$18.2 \mathrm{~J} / \mathrm{cm}^{2}$
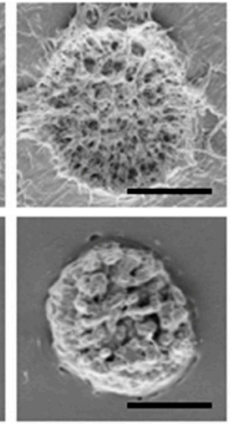

(b)

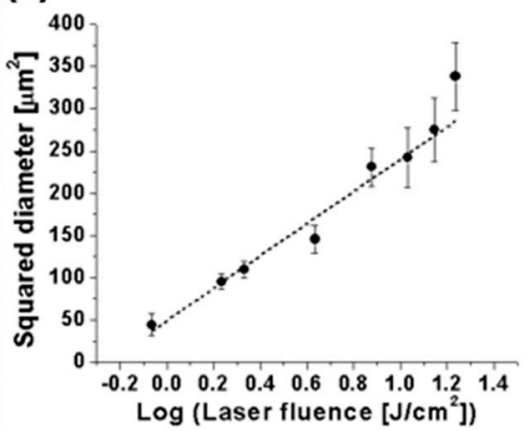

Figure 1. Single laser pulse ablation of polytetrafluoroethylene (PTFE) and process producing numerous polydimethylsiloxane (PDMS) process producing numerous polydimethylsiloxane (PDMS) replica: (a) scanning electron microscopy (SEM) pictures of ablated PTFE surface under varied laser fluence; (b) The squared diameter $\left(D^{2}\right)$ of the ablated PTFE spot is plotted as a function of the laser fluence in log scale; (c) SEM pictures of PDMS replica from the ablated PTFE surface (a). The scale bars are $10 \mu \mathrm{m}$.

We further investigate a scanning scheme for writing line patterns with micro/nano hierarchical surface. Upon laser irradiation, the motorized stage was translated at speed from 1 to $11 \mathrm{~mm} / \mathrm{s}$; the scanning speed of the laser was determined by the overlap between the laser pulses and the respective incident energy density distribution along the ablation line (Figure 2). Note that the repetition rate of the laser was set to $1 \mathrm{kHz}$. Figure 2a shows SEM images of a PTFE surface at the laser fluence of $56 \mathrm{~J} / \mathrm{cm}^{2}$ and the corresponding PDMS replica image. The ablation line width did not depend much on the scanning speed, in contrast to the variation of processed depth. The depth of the ablated concave PTFE structures was directly reflected to the height of convex PDMS replicas as shown in tilted SEM pictures (Figure 2b). Height increase of the PDMS replica was observed as the scanning speed decreased. For example, at the scanning speed of $11 \mathrm{~mm} / \mathrm{s}$ (maximum scan speed in our experiment), the pitch between adjacent laser pulses was $11 \mu \mathrm{m}$, and a train of adjacent single pulse ablation features was observed. On the other hand, at lower scanning speeds these distinctive periodic features could not be seen, and the height of PDMS replicas (or the depth of PTFE template) increased (or deepened) due to the pulse accumulation effect [20]. Therefore, a dramatic height increase was seen at our minimum scanning speed of $1 \mathrm{~mm} / \mathrm{s}$ (or overlapped laser pulses with $1 \mu \mathrm{m}$ pitch). Table 1 summarizes our findings for averaged height of PDMS replica under different laser fluences and scanning speeds of laser. When we magnify laser processed PTFE and PDMS region (marked with circle and box patterns in Figure 2a), the surface of laser ablated micron structure is decorated with nano roughness, resulting in micro/nano hierarchical structures. The magnified SEM pictures confirm that the pulsed laser process allows generation of complex micro/nano hierarchical structures without expensive, complicated, and multi-step processes involving vacuum and mask tools. Because the adhesion strength of the interface between PTFE and PDMS is relatively very low, additional chemical or physical anti-adhesive surface agent treatment is not necessary either; in order to produce a PDMS replica from a surface modified template, anti-adhesive coating is typically required $[25,26]$. 
Table 1. Averaged height ( $\mu \mathrm{m}$ ) of PDMS replica (or averaged depth of PTFE surface) under varied laser fluences and sample scanning speeds.

\begin{tabular}{ccccccc}
\hline & \multicolumn{6}{c}{ Scanning Speeds $(\mathbf{m m} / \mathbf{s})$} \\
\cline { 2 - 7 } Laser Fluence $\left(\mathbf{J} / \mathbf{c m}^{2}\right)$ & $\mathbf{3}$ & $\mathbf{5}$ & $\mathbf{7}$ & $\mathbf{9}$ & $\mathbf{1 1}$ \\
\cline { 2 - 7 } & $\mathbf{1}$ & 12.6 & 7.2 & 6.5 & 4.4 & 3.0 \\
24.3 & 32.0 & 14.0 & 8.2 & 7.0 & 5.5 & 4.0 \\
35.0 & 37.6 & 17.1 & 10.1 & 8.0 & 6.8 & 5.7 \\
45.5 & 40.3 & 18.0 & 11.5 & 8.6 & 7.3 & 6.2 \\
56.0 & & & & & & \\
\hline
\end{tabular}

(a) $1 \mathrm{~mm} / \mathrm{s} 3 \mathrm{~mm} / \mathrm{s} 5 \mathrm{~mm} / \mathrm{s} 7 \mathrm{~mm} / \mathrm{s} 9 \mathrm{~mm} / \mathrm{s} \quad 11 \mathrm{~mm} / \mathrm{s}$
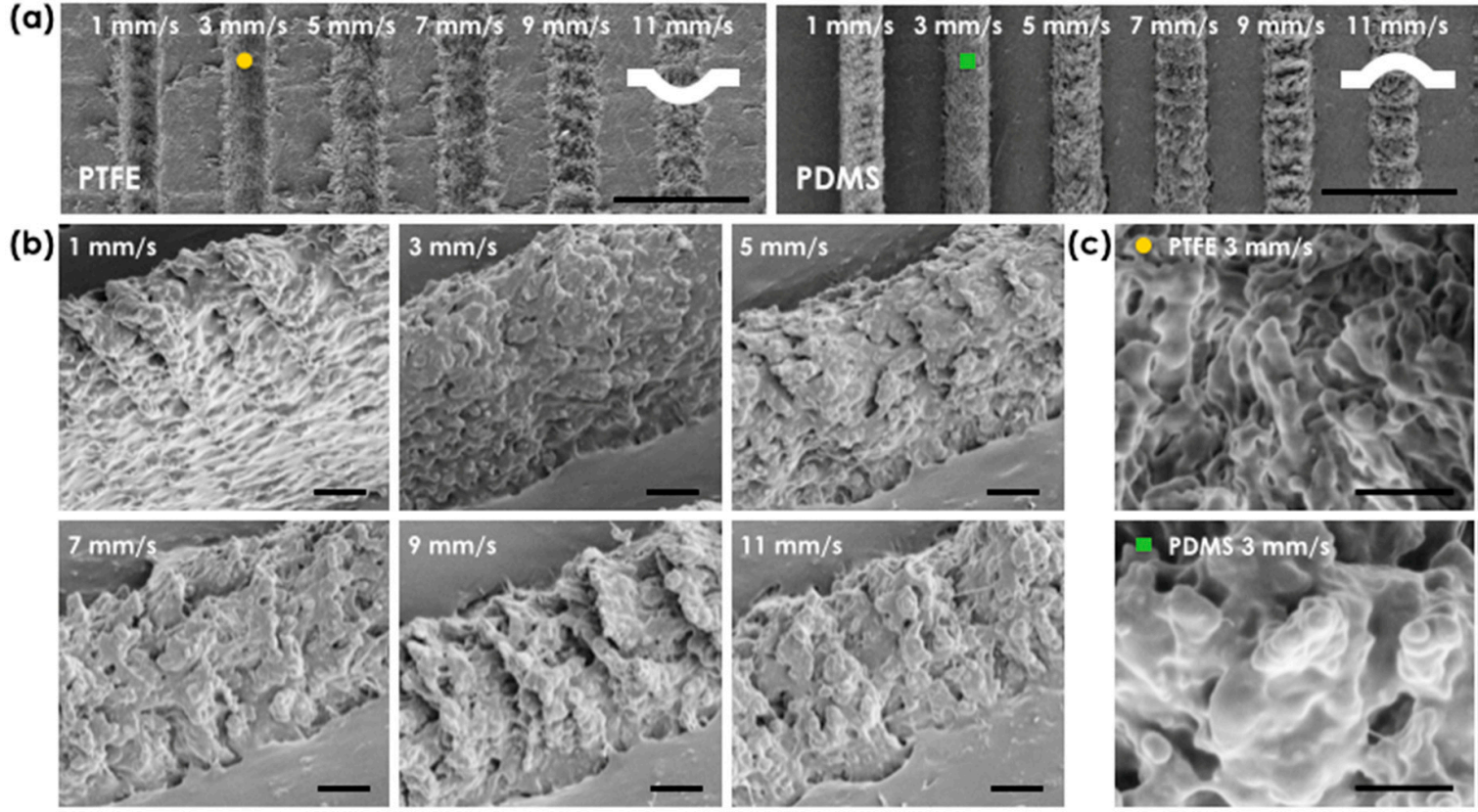

Figure 2. Line ablation with rough surface on a PTFE film by sample scanning: (a) SEM pictures of ablated PTFE film at the laser fluence of $56 \mathrm{~J} / \mathrm{cm}^{2}$ and varied sample scanning speeds from 1 to $11 \mathrm{~mm} / \mathrm{s}$, and PDMS replica from the PTFE film. The scale bars are $50 \mu \mathrm{m}$. The white curved lines illustrate the surface profiles of PTFE and PDMS films; (b) Tilted SEM pictures of the PDMS replica. The scanning speed affects the height of PDMS replica (or the depth of PTFE template). The scale bars are $5 \mu \mathrm{m}$; (c) Magnified SEM pictures of rough PTFE and PDMS region (marking with circle and box in (a)) at the scanning speed of $3 \mathrm{~mm} / \mathrm{s}$. The scale bars are $2 \mu \mathrm{m}$.

In order to control hydrophobicity over a large surface area, we designed mesh patterns of hierarchically structured lines with two design control parameters: pitch $(55,85,130,190,265,355$, and $460 \mu \mathrm{m})$ and scanning speed $(1,3,5,7,9$, and $11 \mathrm{~mm} / \mathrm{s})$. Figure 3 a shows three representative microscopic images of PTFE surfaces having different pitch of 55 (minimum), 190 (medium), and $460 \mu \mathrm{m}$ (maximum) at the fixed scanning speed of $3 \mathrm{~mm} / \mathrm{s}$. In addition, PDMS patterns of the same design parameters were directly replicated from the PTFE surface as shown in the optical microscope images of Figure 3b. Confocal microscopic images of PDMS surface (Figure 3c), showed faithfully transferred convex mesh patterns. 
(a)

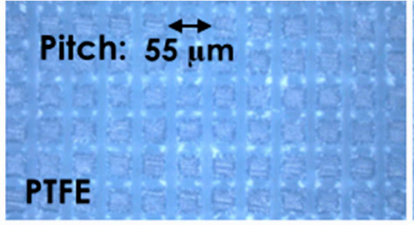

(b)

(c)

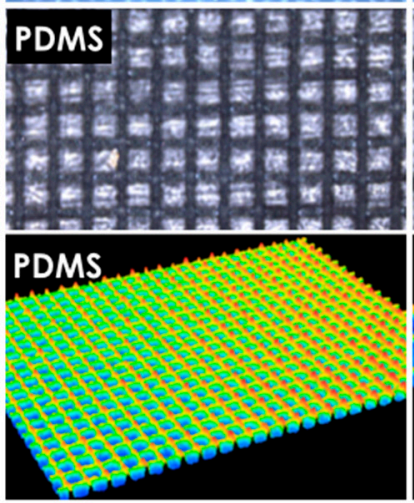

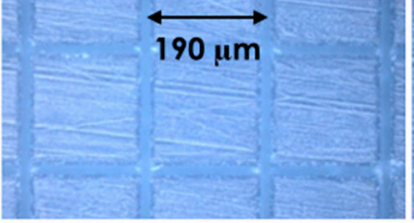
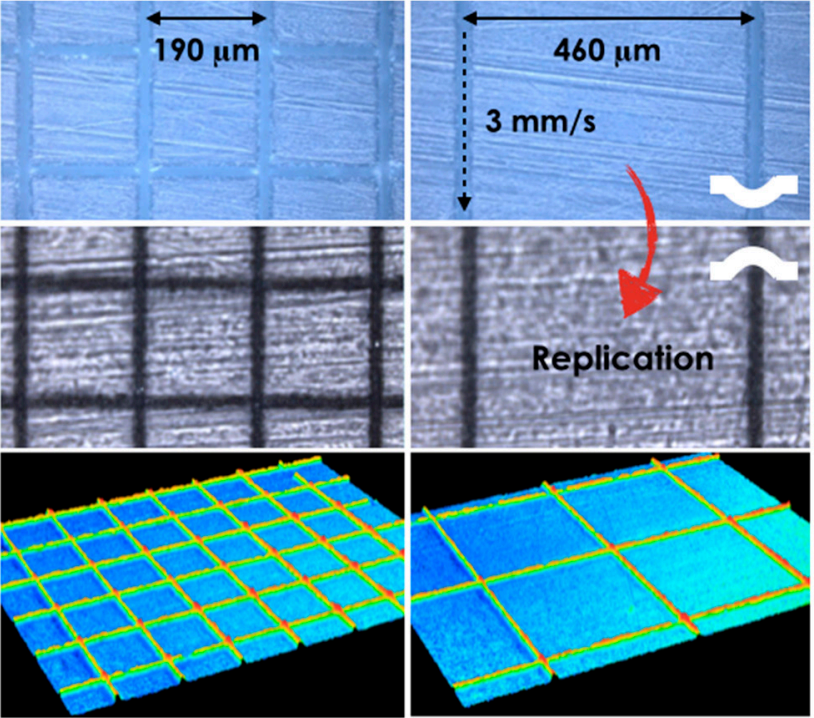

Figure 3. Direct PTFE surface morphology modification and PDMS replica: Microscopic pictures of (a) PTFE surface and (b) PDMS replica with varied pitches of 55, 190, and $460 \mu \mathrm{m}$, at the scanning speed of $3 \mathrm{~mm} / \mathrm{s}$. The white schematics describe the surface morphology; (c) Confocal microscopic pictures of PDMS replica with varied pitches of 55, 190, and $460 \mu \mathrm{m}$, at the scanning speed of $1 \mathrm{~mm} / \mathrm{s}$.

Contact angles of water droplets were measured on the laser-processed PTFE film with the designed patterns as well as on the PDMS replicas (described in Figure 3) and are presented in Figure 4. For the measurement, water droplets with the volume of $\sim 1 \mu \mathrm{L}$ were loaded on both the modified surfaces of PTFE film and the PDMS replica. Since our surfaces are rough with hierarchical structure, the wetting trend can be interpreted by the modified Young's equations, either in the Wenzel model state $\left(\cos \theta^{\prime}=r\left(\gamma_{s v}-\gamma_{s l}\right) / \gamma_{l v}\right.$, where $\theta^{\prime}$ is contact angle, $r$ is the roughness factor, and $\gamma_{s v}, \gamma_{s l}$, and $\gamma_{l v}$, respectively denote the interfacial free energies per unit area of the solid-vapor, solid-liquid, and liquid-vapor interfaces) or in a Cassie-Baxter state $\left(\cos \theta^{\prime}=f \cos \theta+f-1\right.$, where $f$ is an area fraction of wetted solid surface). We should note that Wenzel state (water droplet fills in the cavities or grooves on the surface) is dominant when the surface has small surface roughness, and Cassie's model (air intrudes into the cavities on the surface and make air-water droplet interface) becomes effective as surface roughness increases. The models imply that the parameters $r$ or $f$ (i.e., increasing density of hierarchical structure) should increase in order to obtain high contact angle. Figure $4 \mathrm{a}, \mathrm{b}$ show the side view of droplets placed on the modified surfaces with varied pitches (at the constant laser fluence of $35.0 \mathrm{~J} / \mathrm{cm}^{2}$ and the fixed scanning speed of $3 \mathrm{~mm} / \mathrm{s}$ ) and their contact angles, respectively; the droplet becomes more spherical (i.e., increasing contact angle) as the line pattern pitch is decreased. These results are generally in line with our earlier expectations. However, a closer look revealed that wetting and contact angle exhibited some differences on surface with different surface free energy (PTFE: 18 26 mJ/m² and PDMS: 15 22 mJ/m²) even though surfaces had the same geometry; the contact angle of pristine PTFE and PDMS surface was measured as $107.2^{\circ}$ and $112.1^{\circ}$, respectively, because high free energy surfaces were more easily wet (i.e., low contact angle) than low free energy surfaces. In this respect, super-hydrophobic PDMS replica surface with a relatively low surface free energy compared to PTFE was achieved, when the pitch was 130, 85, and $55 \mu \mathrm{m}$. In contrast, super-hydrophobicity was achieved on PTFE surfaces only when the pitch was $55 \mu \mathrm{m}$. We also measured the contact angles dependence on the scanning speed for the representative three pitches $(55,190$, and $460 \mu \mathrm{m})$ as shown in Figure 4c. The result shows that the contact angle of PTFE and PDMS replica increases as the scan speed decreases. An abrupt increase in the contact angle of PDMS replicas (marked by red dashed arrows in Figure 4c) was observed at the scanning speed of $1 \mathrm{~mm} / \mathrm{s}$, where a dramatic height increase of the hierarchical structure was obtained (see Table 1 in detail). 
This is because the PDMS replica sample has a convex surface; in contrast, the surface morphology of PTFE sample is concave. In the case of PDMS replica, convex protrusions on the surface can induce air pockets and their micro/nano hierarchical structures also can effectively promote the formation of air-trapping regime under the liquid. Moreover, we observed and discussed the large height increase at the scanning speed of $1 \mathrm{~mm} / \mathrm{s}$ (see Figure 2 and Table 1). Therefore, the hydrophobic nature of the surface is further reinforced. On the contrary, in the case of PTFE sample, the micro/nano hierarchical structures cannot be beneficial for the hydrophobicity because the trapped air inside the concavely formed structures stops a drop from going to a place where there are many micro/nano hierarchical structures; a drop just rests on a surface without hierarchical structure, where the interfaces are all planar (composed of solid and air). Therefore, the contact angle was not highly affected by the scanning speed. Moreover, at the small pitch of $55 \mu \mathrm{m}$, the droplets could rest on top of the convex pillars only. At this point, the surface roughness no longer offered advantages with regard to promoting hydrophobicity; there were not much change of contact angles caused by the scan speeds at the pitch of $55 \mu \mathrm{m}$. These results show that we can make large effective super-hydrophobic surface with relatively big pitch $(190 \mu \mathrm{m})$ that suits large-scale processing and related applications.
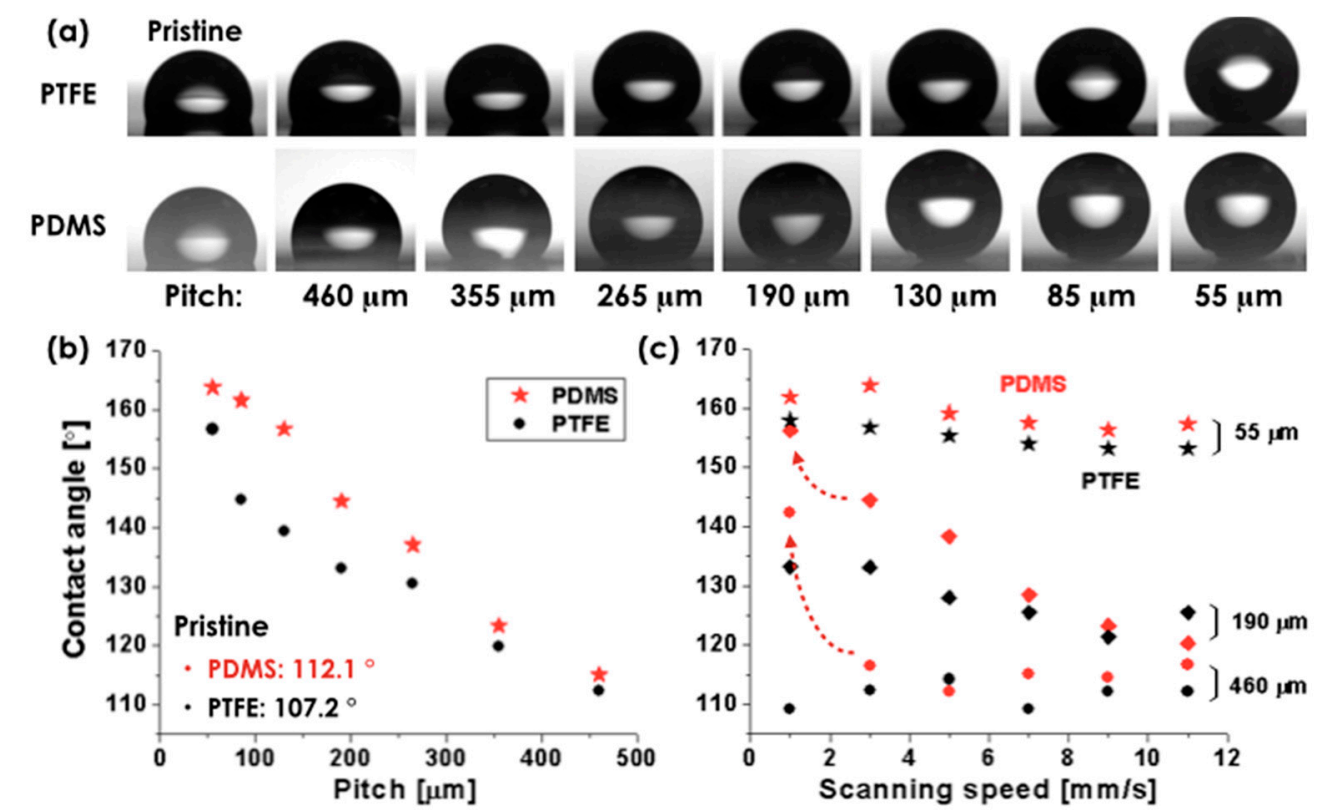

(c) 170

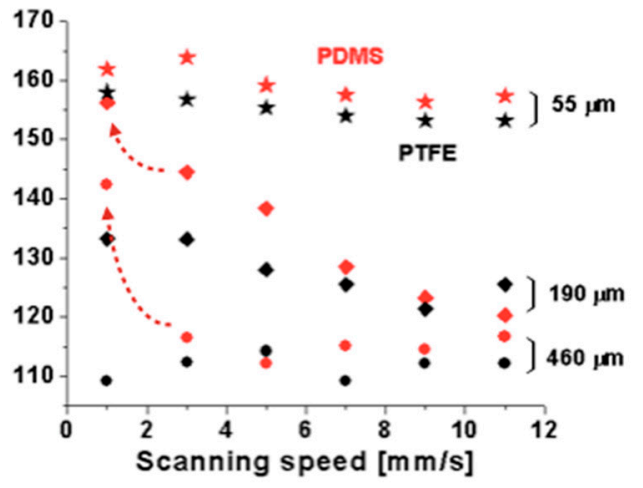

Figure 4. (a) Microscopic pictures of water droplet on surface modified PTFE sample and PDMS replica at the laser fluence of $35.0 \mathrm{~J} / \mathrm{cm}^{2}$ and the scanning speed of $3 \mathrm{~mm} / \mathrm{s} ;(\mathbf{b})$ measured contact angle of the droplets in (a); and (c) contact angle of droplets on surface modified PTFE sample at varied scanning speeds and PDMS replica.

Due to the low adhesion of the water droplet on our super-hydrophobic surface, it is rather difficult to localize a droplet on a specific surface spot by contact using a syringe needle or a micropipette. To overcome this practical issue, we utilized the flexibility of the super-hydrophobic PDMS replica film. To control deformation, we attached the super-hydrophobic PDMS top layer $(5 \mathrm{~cm} \times 5.5 \mathrm{~cm})$ on the pristine PDMS bottom layer with through holes as shown in Figure 5a. Then, a glass vacuum filter holder (Whatman, Maidstone, UK, 1960-004) with $47 \mathrm{~mm}$ diameter was firstly combined on a neck of an opened side arm flask which was connected to a vacuum pump (FJC, Mooresville, NC, USA, 6912). As described in the schematics, the top layer over the hole was pneumatically deformed via a vacuum filtration apparatus. When droplets were dropped on the surface, they self-assembled in the center of the deformed zone (Figure 5a). We note that fluorescence particles (red and yellow) were used to improve the clarity of the droplets and ultraviolet (UV) light helped them to emit their distinct colors. In Figure 5b, we can clearly observe the deformed surface and the site-specifically located droplets 
with fluorescence emission under UV irradiation. Because the single pass laser process produced super-hydrophobic surface with micro/nano hierarchical structures, the shape of the droplets on the surface was approximately spherical.
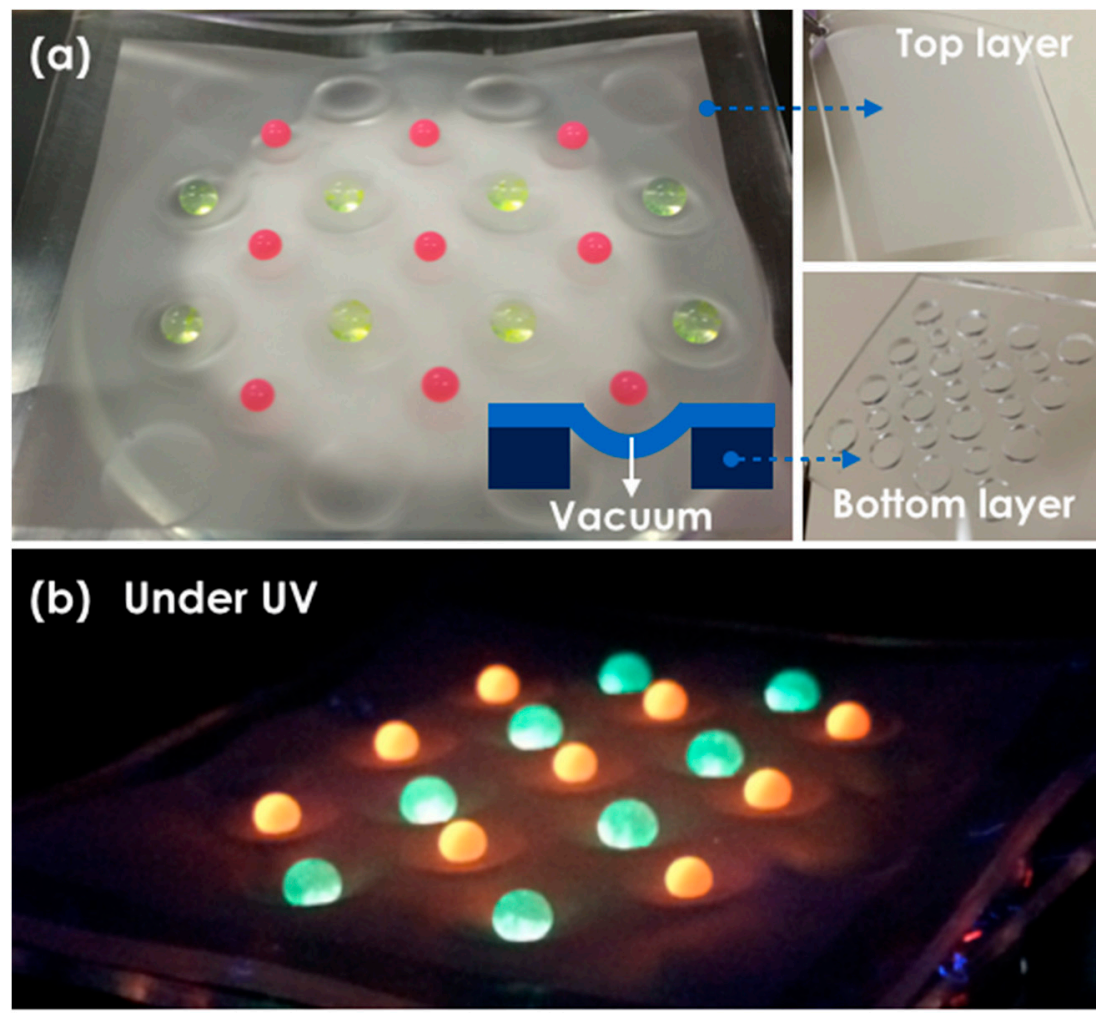

Figure 5. Pneumatically deformed flexible PDMS superhydrophobic film: (a) droplets with fluorescence particles were positioned on the deformed zone. The volumes of the red and yellow droplets were 5 and $10 \mu \mathrm{L}$, respectively; (b) a tilted photograph under UV irradiation.

\section{Conclusions}

In summary, we demonstrated a simple, single pass laser direct writing process for fabricating super-hydrophobic surface without requiring any chemical treatment and additional processes. Femtosecond laser irradiated PTFE film, incurring, absorption via a nonlinear multi-photon process. After the single laser scanning pass (or single shot for a stationary sample), micro/nano hierarchical structures were easily formed on the processed PTFE film. Also, super-hydrophobic PDMS replication was directly achieved from the laser processed PTFE template without requiring anti-adhesive coating treatment because of the anti-adhesive property (i.e., low surface free energy) of PTFE. Moreover, the process can be programmed with designed mesh patterns suitable for a large area. Therefore, we expect the process offers time and cost reduction to form super-hydrophobic flexible surfaces and may emerge as a promising technology.

Author Contributions: Conceptualization, H.-J.K., J.-H.Y., and C.P.G.; Experiments and data analysis, H.-J.K., J.Y., and J.-H.Y.; Investigation, J.Y., J.E.J., and C.P.G.; Writing—Original Draft Preparation, H.-J.K. and J.-H.Y.; Writing-Review \& Editing, J.Y., J.E.J., and C.P.G.

Funding: This work was supported by the DGIST Start-up Fund Program of the Ministry of Science, ICT and Future Planning (2018010064). This work was also performed under the auspices of the U.S. Department of Energy by Lawrence Livermore National Laboratory under Contract No. DE-AC52-07NA27344.

Conflicts of Interest: The authors declare no conflict of interest. 


\section{References}

1. Lafuma, A.; Quere, D. Superhydrophobic states. Nat. Mater. 2003, 2, 457-460. [CrossRef] [PubMed]

2. Nosonovsky, M.; Bhushan, B. Biomimetic superhydrophobic surfaces: Multiscale approach. Nano Lett. 2007, 7, 2633-2637. [CrossRef] [PubMed]

3. Ueda, E.; Levkin, P.A. Emerging Applications of Superhydrophilic-Superhydrophobic Micropatterns. Adv. Mater. 2013, 25, 1234-1247. [CrossRef] [PubMed]

4. Lowe, S.; O’Brien-Simpson, N.M.; Connal, L.A. Antibiofouling polymer interfaces: Poly(ethylene glycol) and other promising candidates. Polym. Chem. 2015, 6, 198-212. [CrossRef]

5. Van der Wal, P.; Steiner, U. Super-hydrophobic surfaces made from Teflon. Soft Matter 2007, 3, 426-429. [CrossRef]

6. Satyaprasad, A.; Jain, V.; Nema, S.K. Deposition of superhydrophobic nanostructured Teflon-like coating using expanding plasma arc. Appl. Surf. Sci. 2007, 253, 5462-5466. [CrossRef]

7. Guo, Z.G.; Liu, W.M.; Su, B.L. Superhydrophobic surfaces: From natural to biomimetic to functional. J. Colloid Interface Sci. 2011, 353, 335-355. [CrossRef] [PubMed]

8. Lee, Y.W.; Park, S.H.; Kim, K.B.; Lee, J.K. Fabrication of hierarchical structures on a polymer surface to mimic natural superhydrophobic surfaces. Adv. Mater. 2007, 19, 2330-2335. [CrossRef]

9. Ariga, K.; Yamauchi, Y.; Rydzek, G.; Ji, Q.M.; Yonamine, Y.; Wu, K.C.W.; Hill, J.P. Layer-by-layer Nanoarchitectonics: Invention, Innovation, and Evolution. Chem. Lett. 2014, 43, 36-68. [CrossRef]

10. Wisdom, K.M.; Watson, J.A.; Qu, X.P.; Liu, F.J.; Watson, G.S.; Chen, C.H. Self-cleaning of superhydrophobic surfaces by self-propelled jumping condensate. Proc. Natl. Acad. Sci. USA 2013, 110, 7992-7997. [CrossRef] [PubMed]

11. Zhang, X.; Guo, Y.G.; Zhang, Z.J.; Zhang, P.Y. Self-cleaning superhydrophobic surface based on titanium dioxide nanowires combined with polydimethylsiloxane. Appl. Surf. Sci. 2013, 284, 319-323. [CrossRef]

12. Chen, Y.; Zhang, Y.B.; Shi, L.; Li, J.; Xin, Y.; Yang, T.T.; Guo, Z.G. Transparent superhydrophobic/superhydrophilic coatings for self-cleaning and anti-fogging. Appl. Phys. Lett. 2012, 101, 033701. [CrossRef]

13. Boinovich, L.B.; Emelyanenko, A.M. Anti-icing potential of superhydrophobic coatings. Mendeleev Commun. 2013, 23, 3-10. [CrossRef]

14. Jung, S.; Dorrestijn, M.; Raps, D.; Das, A.; Megaridis, C.M.; Poulikakos, D. Are Superhydrophobic Surfaces Best for Icephobicity? Langmuir 2011, 27, 3059-3066. [CrossRef] [PubMed]

15. Miljkovic, N.; Enright, R.; Nam, Y.; Lopez, K.; Dou, N.; Sack, J.; Wang, E.N. Jumping-Droplet-Enhanced Condensation on Scalable Superhydrophobic Nanostructured Surfaces. Nano Lett. 2013, 13, 179-187. [CrossRef] [PubMed]

16. Mertaniemi, H.; Jokinen, V.; Sainiemi, L.; Franssila, S.; Marmur, A.; Ikkala, O.; Ras, R.H.A. Superhydrophobic Tracks for Low-Friction, Guided Transport of Water Droplets. Adv. Mater. 2011, 23, 2911-2914. [CrossRef] [PubMed]

17. Hong, X.; Gao, X.F.; Jiang, L. Application of superhydrophobic surface with high adhesive force in no lost transport of superparamagnetic microdroplet. J. Am. Chem. Soc. 2007, 129, 1478-1479. [CrossRef] [PubMed]

18. Accardo, A.; Mecarini, F.; Leoncini, M.; Brandi, F.; Di Cola, E.; Burghammer, M.; Riekel, C.; Di Fabrizio, E. Fast, active droplet interaction: Coalescence and reactive mixing controlled by electrowetting on a superhydrophobic surface. Lab Chip 2013, 13, 332-335. [CrossRef] [PubMed]

19. Manukyan, G.; Oh, J.M.; van den Ende, D.; Lammertink, R.G.H.; Mugele, F. Electrical Switching of Wetting States on Superhydrophobic Surfaces: A Route Towards Reversible Cassie-to-Wenzel Transitions. Phys. Rev. Lett. 2011, 106, 014501. [CrossRef] [PubMed]

20. Athanasiou, C.-E.; Hongler, M.-O.; Bellouard, Y. Unraveling Brittle-Fracture Statistics from Intermittent Patterns Formed During Femtosecond Laser Exposure. Phys. Rev. Appl. 2017, 8, 054013. [CrossRef]

21. Bricchi, E.; Klappauf, B.G.; Kazansky, P.G. Form Birefringence and Negative Index Change Created by Femtosecond Direct Writing in Transparent Materials. Opt. Lett. 2004, 29, 119-121. [CrossRef] [PubMed]

22. Wang, Z.B.; Hong, M.H.; Lu, Y.F.; Wu, D.J.; Lan, B.; Chong, T.C. Femtosecond laser ablation of polytetrafluoroethylene (Teflon) in ambient air. J. Appl. Phys. 2003, 93, 6375-6380. [CrossRef]

23. Kumagai, H.; Midorikawa, K.; Toyoda, K.; Nakamura, S.; Okamoto, T.; Obara, M. Ablation of Polymer-Films by a Femtosecond High-Peak-Power Ti Sapphire Laser at 798-Nm. Appl. Phys. Lett. 1994, 65, 1850-1852. [CrossRef] 
24. Yoo, J.H.; In, J.B.; Park, J.B.; Jeon, H.J.; Grigoropoulos, C.P. Graphene folds by femtosecond laser ablation. Appl. Phys. Lett. 2012, 100, 233124. [CrossRef]

25. Nayak, B.K.; Caffrey, P.O.; Speck, C.R.; Gupta, M.C. Superhydrophobic surfaces by replication of micro/nano-structures fabricated by ultrafast-laser-microtexturing. Appl. Surf. Sci. 2013, 266, 27-32. [CrossRef]

26. Jiang, T.; Koch, J.; Unger, C.; Fadeeva, E.; Koroleva, A.; Zhao, Q.L.; Chichkov, B.N. Ultrashort picosecond laser processing of micro-molds for fabricating plastic parts with superhydrophobic surfaces. Appl. Phys. A-Mater. 2012, 108, 863-869. [CrossRef]

(C) 2018 by the authors. Licensee MDPI, Basel, Switzerland. This article is an open access article distributed under the terms and conditions of the Creative Commons Attribution (CC BY) license (http:/ / creativecommons.org/licenses/by/4.0/). 